\title{
Electrostatic Reconstruction Technology in Atom Probe Tomography
}

Brian Geiser $^{1}$, Isabelle Martin ${ }^{2}$, David Reinhard ${ }^{3}$, Dan Lenz ${ }^{2}$, Ty Prosa ${ }^{4}$, Robert Ulfig ${ }^{3}$ and David Larson $^{3}$

${ }^{1}$ CAMECA Instruments Inc., United States, ${ }^{2}$ CAMECA® Instruments Inc., 5470 Nobel Drive, Madison, WI 53711 USA, United States, ${ }^{3}$ CAMECA Instruments Inc., Madison, Wisconsin, United States, ${ }^{4}$ CAMECA® Instruments Inc., 5470 Nobel Drive, Madison, WI 53711 USA, Wisconsin, United States

In atom probe tomography (APT) a primary element of the instrument optics is the specimen itself. In order to correct artifacts in the data caused by an evolving apex shape, we are developing a numerical electrostatic model $[1,2]$ reflecting the physics of both the instrumental pieces as well as the specimen geometry. In this poster we report on some numerical accuracy metrics of this model and illustrate its use in the generation of simulated evaporation data.

For the accuracy tests, we consider a spherical apex (Fig. 1a). To model the potentials, we mesh the apex with concentric rings of charges, the magnitude of which are chosen to produce prescribed potentials at prescribed sensor positions on the various surfaces (Fig. 1b). After computing the charges, we propagate trajectories from all the specified sensor positions on the apex to a model detector plane. Due to the complicated fields around the specimen, we don't know the actual correct hit positions, but since the model is designed to be axially symmetric we can make two self-consistency measurements: 1) dispersion in hit position radius for all trajectories generated from a single ring (Fig. 1c), and 2) deviation in the expected lateral position given the angular position of a trajectory launch position (Fig. 1d).

This full-instrument optical model also gives us the opportunity to improve the usefulness of our evaporation simulation system. Previously, TipSim [3] simulated an evaporation volume with a 3d grid that evolved under a finite difference potential calculation. Even with nested volumes, (Fig. 2a) that scale in size, it is difficult to scale up the system to a point where it could embody both the full specimen geometry and a local electrode [4]. We now implement the hybrid system shown in Fig. 2b, where ions are evaporated in the finite difference grid, propagate through it and then propagate through the distributed point source method (DPSM) model for several hundred microns. After that distance we then propagate to the detector plane by using a precalculated transfer function specific to different instrument models.

1a)

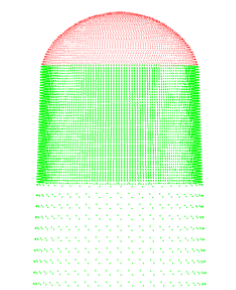

1b)

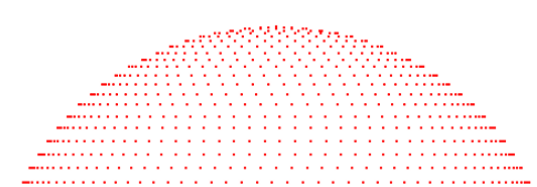

1c)

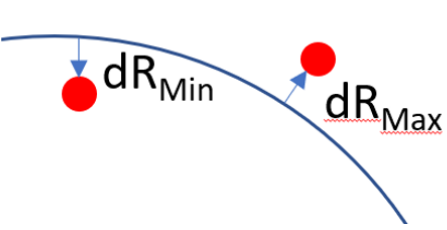

1d)

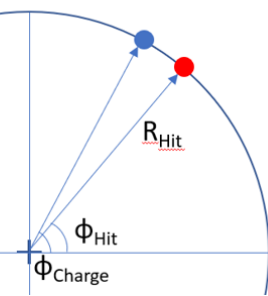

Figure 1. a) The apex and near-shank section of a charge-model. b) A close-up of the apex rings of charge. c) The measurement of dispersion in detected hit radius. d) The measurement of deviation in expected hit lateral position. 

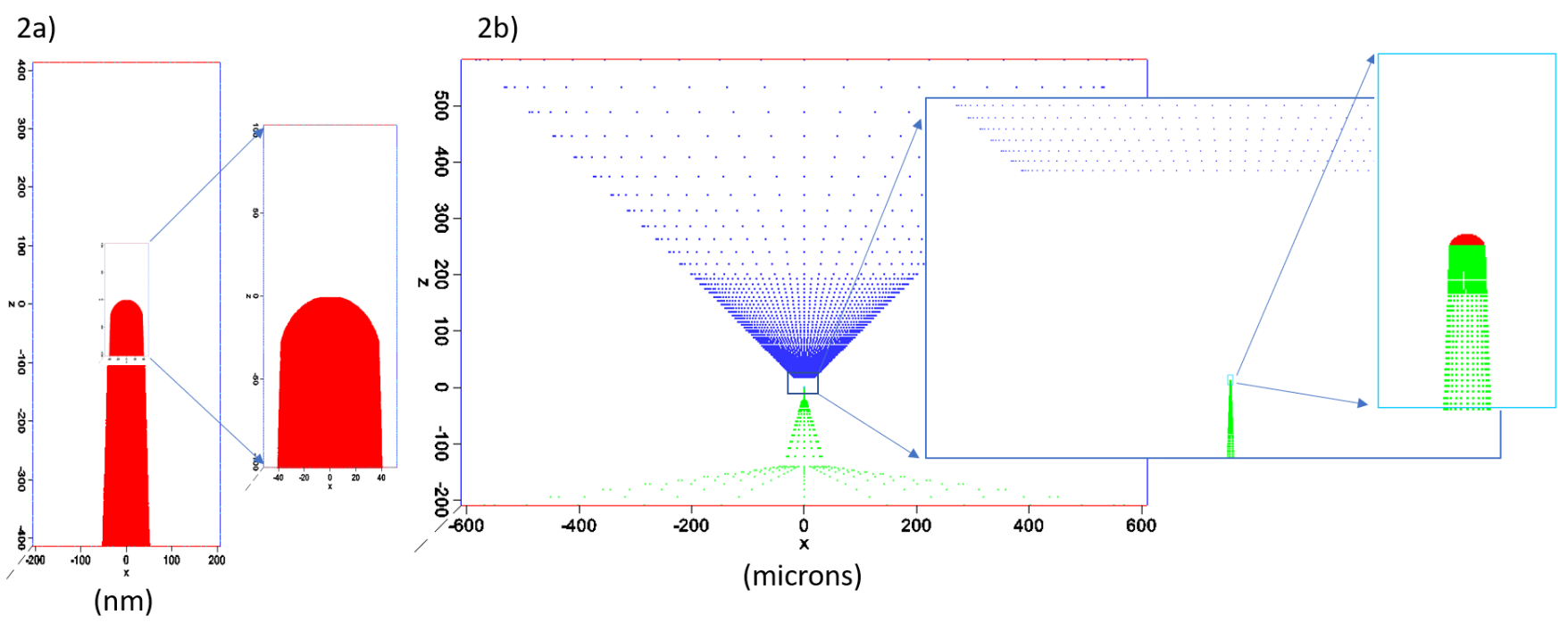

Figure 2. a) Two nested grid volumes for evaporation simulation. b) The larger nesting volume is shown in the context of the charge model.

\section{References}

[1] B. Geiser et al., Micro. Microanal. 26(2) (2020) p. 2622.

[2] D. Placko and T. Kundu, eds. DPSM for Modeling Engineering Problems. John Wiley \& Sons, (2007).

[3] B. Geiser et al., Micro. Microanal. 15(2) (2009) p. 302.

[4] D. J. Larson et al., "Local Electrode Atom Probe Tomography: A User's Guide”, (Springer Scientific, New York, 2013). 